\title{
Two kinds of orthopair soft sets as novel approaches to granular computing based on parametrization
}

\author{
M.I. Ali ${ }^{1}$, M. Shabir ${ }^{2}$ and F. Feng ${ }^{3}$ \\ ${ }^{1}$ Islamabad Model College for Boys F-10/3, Islamabad, Pakistan \\ ${ }^{2}$ Department of Mathematics, Quaid-i-Azam University, Islamabad, Pakistan \\ ${ }^{3}$ School of Science, Xi'an University of Posts and Telecommunications, Xi'an, China \\ mirfanali13@yahoo.com, mshabirbhatti@yahoo.co.uk, fengnix@hotmail.com
}

"This paper is dedicated to Professor Young Bae Jun on the occasion of his 70th birthday."

\begin{abstract}
This paper aims at introducing two types of orthopair soft sets, which might serve as novel approaches to granular computing based on parametrization. These generalized soft sets emerge naturally when linguistic parameters are employed to convey uncertainty attached to elements of certain sets. Concepts of uncertainty measures attached to the parameters and the whole orthopair soft sets are presented as well. The proposed uncertainty measures are useful for classifying elements of the set of parameters. Different types of granularity measures associated with parameters are presented and are extended to orthopair soft sets. Collective wisdom is helpful in decision making based on consensus. A numerical example is given to demonstrate how orthopair soft sets can be employed in this regard.
\end{abstract}

\section{Article Information}

Corresponding Author:

F. Feng;

Received: June 2021;

Accepted: Invited paper;

Paper type: Original.

\section{Keywords:}

Soft sets, fuzzy sets, rough sets, orthopair, uncertainty, granularity.

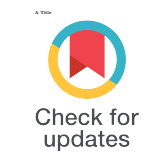

\section{Introduction}

In human life uncertainty is abundantly attached to daily information. Uncertainty may be due to lack of information, granularity and difference of opinion. Many approaches including fuzzy sets [31], shadowed sets [22, 23, 24], rough sets [20, 21] and soft sets [18] have been introduced to deal with different kinds of uncertainties. Many generalizations of above mentioned theories are now available to take much complicated situations. Zadeh's fuzzy set theory is an attempt to deal with uncertainty caused by difference of opinion. Pawlak's rough set theory considers the uncertainty

https://doi.org/10.52547/HATEF.JAHLA.2.3.4 
caused by the granularity. In Molodtsov's soft set theory, there are some parameters available to tackle uncertain situations. Many applications of soft sets have been reported in [3, 4, 6, [25].

Notion of flou sets was introduced in [16]. A flou set is an ordered pair $(X, Y)$ such that $X \subseteq Y$. According to [30], flou sets are also known as interval sets. In Pawlak's rough set model [201, 21] , such pairs arise in a natural way when lower and upper approximations of a set are obtained. It is evident that if we consider the pair $\left(X, Z^{c}\right)$, where $X \subseteq Z$, then $X \cap Z^{c}=\emptyset$. Generally, name of orthopair sets is given to such pairs $(X, Y)$ where $X \cap Y=\emptyset$. This means there exists a bijection between orthopair sets and flou sets but difference of their semantics compel to define their basic operations such as union and intersection in distinct ways. This difference has been pointed out in [10] by Ciucci.

In [10], an inspiring discussion is given to explain two types of above mentioned orthopair sets. Relationship between orthopair sets and other models dealing with uncertainty such as threevalued logic, rough sets, two-fold sets, shadowed sets and conditional events has been established. Bipolarity of information is ubiquitous in both nature and human society. It is considered that processing of such positive and negative information takes place in different parts of a human brain [I2]. In general, when elements of a set are kept as positive then the negative set is not necessarily its complement. Set difference of these two sets is the collection of neutral or uncertain elements. Representation of bipolar information has been discussed in [1, ए2, 1.9]. Generalizations of fuzzy sets to their orthopair counterparts have emerged in a natural way to discuss uncertainty in real-life situations. These include Atanassov's intuitionistic fuzzy sets [ $8, \overline{9}, \mathbf{9}$, Pythagorean fuzzy sets [26, 2.9, 27], q-rung orthopair fuzzy sets [2, 15, 28] and bipolar fuzzy sets [32]. Maji et al. [I7] combined intuitionistic fuzzy sets with soft sets to come up with the notion of intuitionistic fuzzy soft sets. Feng et al. [14] presented a new extension of the preference ranking organization method for enrichment evaluation (PROMETHEE), by taking advantage of intuitionistic fuzzy soft sets. Agarwal et al. [I] further proposed the notion of generalized intuitionistic fuzzy soft sets. Feng et al. [13] clarified and improved the concept of generalized intuitionistic fuzzy soft sets by simplifying it as a combination of an intuitionistic fuzzy soft set over the universe of discourse and an intuitionistic fuzzy set in the parameter set. Although above mentioned orthopairs are very handy to deal with daily life problems yet their application by an ordinary user is not an easy task. The main reason for this drawback is difficulty in definition of membership and nonmembership functions, because it needs expertise in certain areas. Apparently, ordinary people do not need such high level knowledge, therefore they handle uncertainty with the help of properties, attributes or parameters associated with the elements of a set. There are two basic approaches, in first one, elements of a set which surely satisfy a property are considered in first set and the elements which possibly satisfy the same property are collected in the second set of an ordered pair of sets. In second method elements which surely satisfy a property are put together in the first set whereas the elements which surely do not satisfy a given property are collected in the second set.

In this paper, idea of orthopair soft sets and their operations has been introduced, which can be very helpful not only to common people but also for the experts. Here two types of orthopair soft sets are given, which provide parametric representation to both type of above mentioned ordered pairs of sets.

Sequel of the paper is organized as follows. In Section 2 , some important notions related to soft sets and rough sets are given. The concept named orthopair soft sets of first kind is proposed and investigated in Section B. Uncertainty measures for orthopair soft sets of first kind are studied in Section 1 . In Section 15, different types of granularity measures have been given. The notion called orthopair soft sets of second kind and their operations are given in Section 6. For orthopair soft sets of second kind, uncertainty measures are developed in Section $\square$. Section $\mathbb{\nabla}$ deals with different 
kinds of granularity measures for orthopair soft sets of second kind. In Section Q1, a simple example is given to demonstrate how orthopair soft sets can be helpful in the decision making process based on collective wisdom. Finally, we give a summary of our work in Section एu.

\section{$2 \quad$ Preliminaries}

In this section, some fundamental concepts related to this work are considered. Throughout this study, $U$ will denote a nonempty finite set unless stated otherwise. The power set of $U$ will be written as $\mathcal{P}(U)$. Notion of a soft set is introduced by Molodtsov [18] as the following.

Let $E$ be the parameter space and $A \subseteq E$. Then a pair $(F, A)$ is called a soft set over $U$, where $F$ is a mapping given by $F: A \rightarrow \mathcal{P}(U)$. For details related to operations of soft sets, please refer to $[$ [5, []].

Rough set theory is presented by Pawlak [20, 2I]].

If $\sigma$ is an equivalence relation on $U$, then $(U, \sigma)$ is called an approximation space. If $X \subseteq U$, then $X$ can be approximated by two subsets called lower and upper approximations of $X$ as the following:

$$
\begin{aligned}
& \underline{\operatorname{apr}(X)}=\cup\left\{[x]_{\sigma}:[x]_{\sigma} \subseteq X\right\} \\
& \overline{\operatorname{apr}}(X)=\cup\left\{[x]_{\sigma}:[x]_{\sigma} \cap X \neq \emptyset\right\} .
\end{aligned}
$$

Note that $[x]_{\sigma}$ is the equivalence class obtained by equivalence relation $\sigma$ containing some element $x \in U$. A set $X$ is called a rough set if $\overline{\operatorname{apr}}(X)-\operatorname{apr}(X) \neq \emptyset$, otherwise it may be termed as a definable set. It is evident from the definition that $\operatorname{apr}(X) \subseteq X \subseteq \overline{\operatorname{apr}}(X)$. If $\operatorname{apr}(X) \neq \emptyset$, then its elements surely belong to $X$, whereas elements of $\overline{a p r}(X)$ are possibly in $X$. If $(\overline{a p r}(X))^{c} \neq \emptyset$, then this set contains those elements which certainly do not belong to $X$.

\section{Orthopair soft sets of first kind}

In this section notion of orthopair soft sets of first kind is introduced. In rough set theory [20, [21], lower approximation of a set is always contained in upper approximation. The difference of upper approximation and lower approximation provides the set of uncertain elements. Here parametric collection of those pairs $(X, Y)$ of sets is being considered for which $X \subseteq Y$.

Definition 3.1. Let $E$ be the parameter space and $A \subseteq E$. An orthopair soft set $(F, A)$ of first kind over a set $U$ is defined by the map $F: A \rightarrow P(U) \times P(U)$ such that $F(\alpha)=(X, Y)$ and $X \subseteq Y$, for all $\alpha \in A$.

This means an orthopair soft set of first kind is a parameterized collection of pair of subsets in $U$. Here the first component $X$ is the necessary part whereas the second component $Y$ is the sufficient part.

An orthopair soft set $(F, A)$ of first kind over a set $U$ reduces to a soft set when $F(\alpha)=(X, Y)$ and $X=Y$ for all $\alpha \in A$.

As for all $\alpha \in A, F(\alpha)=(X, Y)$, therefore each $F(\alpha)$ can be denoted by $\left(X_{F(\alpha)}, Y_{F(\alpha)}\right)$.

Let $O S F(U)$ denote the class of all orthopair soft sets of first kind over $U$.

Definition 3.2. Let $(F, A) \in O S F(U)$. For any $\alpha \in A, F(\alpha)$ may be called a soft element of first kind for $(F, A)$. 
Let $F(\alpha)=\left(X_{F(\alpha)}, Y_{F(\alpha)}\right)$ and $F(\beta)=\left(X_{F(\beta)}, Y_{F(\beta)}\right)$ be two soft elements of first kind for $(F, A) \in O S F(U)$. Then $F(\alpha) \preceq F(\beta)$ if and only if $X_{F(\alpha)} \subseteq X_{F(\beta)}$ and $Y_{F(\alpha)} \subseteq Y_{F(\beta)}$.

Definition 3.3. Let $(F, A) \in O S F(U)$. Then there is an associated boundary soft set denoted by $B N D(F, A)=(B N D(F), A)$ and defined as $B N D(F(\alpha))=Y-X$, for all $\alpha \in A$.

The soft set $B N D(F, A)$ is the parametrized collection of those elements for which it is not sure to satisfy properties associated with parameter $\alpha$, for all $\alpha \in A$.

In order to explain the notion of orthopair soft sets of first kind, the following example may be considered.

Example 3.4. Let us consider a set $U=\left\{c_{1}, c_{2}, c_{3}, c_{4}, c_{5}, c_{6}, c_{7}, c_{8}, c_{9}, c_{10}\right\}$ of companies working in a stock exchange in energy sector. An investor invests in those companies which bring high profits for him/her. It is very difficult to predict about share value of a certain company on a particular day of business. However, considering previous performance of companies share prices, it may be predicted that on a particular day which company will show profit surely or possibly. Let $A=\left\{\alpha_{1}, \alpha_{2}, \alpha_{3}\right\}$ be the set of parameters to denote companies share prices on a particular day. Let $\alpha_{1}$ stands for companies in profit, $\alpha_{2}$ for companies in stable position and $\alpha_{3}$ for companies in loss. An orthopair soft set $(F, A)$ of first kind can be defined as to predict companies performances as follows.

$$
\begin{aligned}
& F\left(\alpha_{1}\right)=\left(\left\{c_{1}, c_{7}, c_{8}\right\},\left\{c_{1}, c_{2}, c_{7}, c_{8}, c_{9}\right\}\right), \\
& F\left(\alpha_{2}\right)=\left(\left\{c_{2}, c_{6}, c_{9}\right\},\left\{c_{2}, c_{3}, c_{4}, c_{6}, c_{8}, c_{9}, c_{10}\right\}\right), \\
& F\left(\alpha_{3}\right)=\left(\left\{c_{3}, c_{4}, c_{5}\right\},\left\{c_{2}, c_{3}, c_{4}, c_{5}, c_{6}, c_{8}, c_{9}, c_{10}\right\}\right) .
\end{aligned}
$$

The associated boundary soft set is as follows:

$\left.B N D(F, A)=B N D F\left(\alpha_{1}\right)=\left\{c_{2}, c_{9}\right\}, B N D F\left(\alpha_{2}\right)=\left\{c_{3}, c_{4}, c_{8}, c_{10}\right\}, B N D F\left(\alpha_{3}\right)=\left\{c_{2}, c_{6}, c_{8}, c_{9}, c_{10}\right\}\right\}$.

Definition 3.5. Let $(F, A),(G, B) \in O S F(U)$. Then $(F, A)$ is an orthopair soft subset of $(G, B)$ if the following hold:

1. $A \subseteq B$.

2. $F(\alpha) \preceq G(\alpha)$ for all $\alpha \in A$.

If $(F, A)$ is an orthopair soft subset of $(G, B)$, then it will be denoted as $(F, A) \widehat{\subseteq}(G, B)$.

Two orthopair soft sets of first kind $(F, A)$ and $(G, B)$ will be equal if $(F, A) \widehat{\subseteq}(G, B)$ and $(G, B) \widehat{\subseteq}(F, A)$.

Let $F(\alpha)=(X, Y)$ be a soft element of first kind for $(F, A) \in O S F(U)$, then $F^{c}(\alpha)=$ $\left(Y_{F(\alpha)}^{c}, X_{F(\alpha)}^{c}\right)$.

Definition 3.6. Let $(F, A) \in O S F(U)$. Then the complement of $(F, A)$ is denoted by $(F, A)^{c}=$ $\left(F^{c}, A\right)$ and is defined as $F^{c}(\alpha)=\left(Y_{F(\alpha)}^{c}, X_{F(\alpha)}^{c}\right)$, where $F(\alpha)=(X, Y)$ for all $\alpha \in A$.

It can be seen that $\left((F, A)^{c}\right)^{c}=(F, A)$.

The orthopair soft set $(\emptyset, A)$ is denoted $\emptyset_{A}$ and is defined as $\emptyset(\alpha)=(\emptyset, \emptyset)$ for all $\alpha \in A$. On the other hand, the orthopair soft set $(U, A)$ is denoted $U_{A}$ and is defined as $U(\alpha)=(U, U)$ for all $\alpha \in A$. In case if $A=\emptyset$, then the orthopair soft set with empty set of parameters will be denoted by $\emptyset_{\emptyset}$. The orthopair soft set $(F, A)$ such that $F(\alpha)=(\emptyset, \emptyset)$ for all $\alpha \in A$, represent the situation 
when it is not possible to select any element of the set $U$ for the parameters $\alpha$. If $(F, A)$ is an orthopair soft set of first kind over $U$, then the following hold

$$
\emptyset_{\emptyset} \widehat{\subseteq} \emptyset_{A} \widehat{\subseteq}(F, A) \widehat{\subseteq}(U, A) .
$$

Note: Complement of $\emptyset_{\emptyset}$ will remain as $\emptyset_{\emptyset}$ because the set of parameters does not change in both cases.

Definition 3.7. Let $(F, A),(G, B) \in O S F(U)$. Then

1. their restricted intersection $(F, A) \cap_{R}(G, B)$ is defined as

$$
(F, A) \cap_{R}(G, B)=(K, C) \text {, where } C=A \cap B .
$$

and when $C \neq \emptyset$

$$
K(\alpha)=\left(X_{F(\alpha)} \cap X_{G(\alpha)}, Y_{F(\alpha)} \cap Y_{G(\alpha)}\right) \text { for all } \alpha \in C,
$$

otherwise $(K, C)=\emptyset_{\emptyset}$.

2. their extended intersection $(F, A) \cap_{\mathcal{E}}(G, B)$ is defined as

$$
(F, A) \cap_{\mathcal{E}}(G, B)=(K, C) \text {, where } C=A \cup B .
$$

and

$$
K(\alpha)=\left\{\begin{array}{cc}
\left(X_{F(\alpha)}, Y_{F(\alpha)}\right) & \text { if } \alpha \in A-B \\
\left(X_{G(\alpha)}, Y_{G(\alpha)}\right) & \text { if } \alpha \in B-A \\
\left(X_{F(\alpha)} \cap X_{G(\alpha)}, Y_{F(\alpha)} \cap Y_{G(\alpha)}\right) & \text { if } \alpha \in A \cap B
\end{array}\right.
$$

3. their restricted union $(F, A) \cup_{R}(G, B)$ is defined as

$$
(F, A) \cup_{R}(G, B)=(K, C) \text {, where } C=A \cap B .
$$

and when $C \neq \emptyset$

$$
K(\alpha)=\left(X_{F(\alpha)} \cup X_{G(\alpha)}, Y_{F(\alpha)} \cup Y_{G(\alpha)}\right) \text { for all } \alpha \in C,
$$

otherwise $(K, C)=\emptyset_{\emptyset}$.

4. their extended union $(F, A) \cup_{\mathcal{E}}(G, B)$ is defined as

$$
(F, A) \cup_{\mathcal{E}}(G, B)=(K, C) \text {, where } C=A \cup B .
$$

and

$$
K(\alpha)=\left\{\begin{array}{cc}
\left(X_{F(\alpha)}, Y_{F(\alpha)}\right) & \text { if } \alpha \in A-B \\
\left(X_{G(\alpha)}, Y_{G(\alpha)}\right) & \text { if } \alpha \in B-A \\
\left(X_{F(\alpha)} \cup X_{G(\alpha)}, Y_{F(\alpha)} \cup Y_{G(\alpha)}\right) & \text { if } \alpha \in A \cap B
\end{array}\right.
$$

Theorem 3.8. Let $(F, A),(G, B),(H, C) \in O S F(U)$. Then we have

$$
((F, A) \theta(G, B)) \theta(H, C)=(F, A) \theta((G, B) \theta(H, C)),
$$

where $\theta \in\left\{\cap_{R}, \cup_{R}, \cap_{\mathcal{E}}, \cup_{\mathcal{E}}\right\}$. 
Theorem 3.9. Let $(F, A),(G, B) \in O S F(U)$. Then the following hold

1. $\left((F, A) \cap_{R}(G, B)\right)^{c}=(F, A)^{c} \cup_{R}(G, B)^{c}$,

2. $\left((F, A) \cup_{R}(G, B)\right)^{c}=(F, A)^{c} \cap_{R}(G, B)^{c}$.

Proof. (1) If $A \cap B=\emptyset$, then equality holds trivially. Let $A \cap B \neq \emptyset$ and $(F, A) \cap_{B}(G, B)=(H, C)$, where $C=A \cap B$ and $H(\alpha)=F(\alpha) \cap G(\alpha)$ for all $\alpha \in C$. As $\left((F, A) \cap_{R}(G, B)\right)^{c}=(H, C)^{c}=$ $\left(H^{c}, C\right)$, where

$$
\begin{aligned}
H^{c}(\alpha) & =(F(\alpha) \cap G(\alpha))^{c} \text { for all } \alpha \in C \\
& =\left(X_{F(\alpha)} \cap X_{G(\beta)}, Y_{F(\alpha)} \cap Y_{G(\beta)}\right)^{c} \\
& =\left(\left(Y_{F(\alpha)} \cap Y_{G(\beta)}\right)^{c},\left(X_{F(\alpha)} \cap X_{G(\beta)}\right)^{c}\right) \\
& =\left(Y_{F(\alpha)}^{c} \cup Y_{G(\beta)}^{c}, X_{F(\alpha)}^{c} \cup X_{G(\beta)}^{c}\right) .
\end{aligned}
$$

On the other hand let $(F, A)^{c} \cup_{R}(G, B)^{c}=(K, C)$. This implies $(K, C)=\left(F^{c}, A\right) \cup_{R}\left(G^{c}, B\right)$. Now for all $\alpha \in C$,

$$
\begin{aligned}
K(\alpha) & =F^{c}(\alpha) \cup G^{c}(\alpha) \\
& =\left(Y_{F(\alpha)}^{c}, X_{F(\alpha)}^{c}\right) \cup\left(Y_{G(\beta)}^{c}, X_{G(\beta)}^{c}\right) \\
& =\left(Y_{F(\alpha)}^{c} \cup Y_{G(\beta)}^{c}, X_{F(\alpha)}^{c} \cup X_{G(\beta)}^{c}\right) .
\end{aligned}
$$

This shows that for all $\alpha \in C, H^{c}(\alpha)=K(\alpha)$. Hence

$$
\left((F, A) \cap_{R}(G, B)\right)^{c}=(F, A)^{c} \cup_{R}(G, B)^{c} .
$$

(2) The proof follows from (1) and thus omitted.

\section{$4 \quad$ Uncertainty measures}

In this section notions related to uncertainty measures will be studied. Study of uncertainty measures can be made under two different but related subsections. Firstly, we will present notions related uncertainty measures for parameters. Secondly, concepts of uncertainty measures for the whole soft set will be given.

\subsection{Uncertainty measures for each parameter of the orthopair soft sets}

Uncertainty measures for a parameter show how clearly objects can be identified with respect to a certain parameter. This notion can be defined as follows.

Definition 4.1. Let $(F, A) \in O S F(U)$. Then for each $\alpha \in A$, an uncertainty measure called uncertainty index for the parameter $\alpha$, denoted by $\pi(\alpha)$, can be defined as

$$
\pi(\alpha)=\frac{|B N D(F(\alpha))|}{|U|}=\frac{\left|Y_{F(\alpha)}-X_{F(\alpha)}\right|}{|U|} .
$$

It is clear that $0 \leq \pi(\alpha) \leq 1$. 
If $F(\alpha)=(\emptyset, U)$, then $\pi(\alpha)=1$. On the contrary, $\pi(\alpha)=0$ when $F(\alpha)=(X, X)$.

Another uncertainty measure, so called associated uncertainty measure can be defined as the following.

Definition 4.2. Let $(F, A) \in O S F(U)$. For each $\alpha \in A$, associated uncertainty index denoted by $\pi^{\prime}(\alpha)$ can be defined as

$$
\pi^{\prime}(\alpha)=\frac{|B N D(F(\alpha))|}{\left|Y_{F(\alpha)}\right|}=\frac{\left|Y_{F(\alpha)}-X_{F(\alpha)}\right|}{\left|Y_{F(\alpha)}\right|} .
$$

It can be seen that $0 \leq \pi^{\prime}(\alpha) \leq 1$.

Proposition 4.3. Let $(F, A) \in O S F(U)$. Then for each $\alpha \in A, \pi(\alpha) \leq \pi^{\prime}(\alpha)$.

Proof. Since $\left|Y_{F(\alpha)}\right| \leq|U|$, we get $\frac{1}{|U|} \leq \frac{1}{\left|Y_{F(\alpha)}\right|}$. This implies that $\frac{\left|Y_{F(\alpha)}-X_{F(\alpha)}\right|}{|U|} \leq \frac{\left|Y_{F(\alpha)}-X_{F(\alpha)}\right|}{\left|Y_{F(\alpha)}\right|}$ that is $\pi(\alpha) \leq \pi^{\prime}(\alpha)$.

Example 4.4. (continued from Example [3.4) It can be seen that $\pi\left(\alpha_{1}\right)=0.2, \pi\left(\alpha_{2}\right)=0.4$, $\pi\left(\alpha_{1}\right)=0.5$. Also $\pi^{\prime}\left(\alpha_{1}\right)=0.4, \pi^{\prime}\left(\alpha_{2}\right)=0.57143, \pi^{\prime}\left(\alpha_{3}\right)=0.625$. Moreover $\pi\left(\alpha_{i}\right) \leq \pi^{\prime}\left(\alpha_{i}\right)$ for $i=1,2,3$.

It is interesting to see that above defined two uncertainty measures induce two equivalence relations on the set of parameters $A$. For this consider

Definition 4.5. Let $(F, A) \in O S F(U)$. For any $\alpha, \beta \in A$, we define

$$
\begin{gathered}
\alpha \sim_{\pi} \beta \quad \Leftrightarrow \quad \pi(\alpha)=\pi(\beta) ; \\
\alpha \sim_{\pi^{\prime}} \beta \quad \Leftrightarrow \quad \pi^{\prime}(\alpha)=\pi^{\prime}(\beta) .
\end{gathered}
$$

It is easy to see that both $\sim_{\pi}$ and $\sim_{\pi^{\prime}}$ are equivalence relations. These two equivalence relations classify parameters in the set $A$ with respect to a particular uncertainty measure.

Let us denote the class containing an element $\alpha \in A$, by $[\alpha]_{\sim_{\pi}}$. Then $A / \sim_{\pi}=\left\{[\alpha]_{\sim_{\pi}}: \alpha \in A\right\}$. Similarly $A / \sim_{\pi^{\prime}}=\left\{[\alpha]_{\sim_{\pi^{\prime}}}: \alpha \in A\right\}$. The following example shows that $\sim_{\pi}$ and $\sim_{\pi^{\prime}}$ are two distinct equivalence relations.

Example 4.6. Let $U=\left\{a_{1}, a_{2}, \ldots, a_{10}\right\}$ be a set and $A=\left\{\alpha_{1}, \alpha_{2}, \alpha_{3}, \alpha_{4}\right\}$ be a set of parameters. An orthopair soft set $(F, A)$ of first kind is defined as the following.

$$
\begin{aligned}
& F\left(\alpha_{1}\right)=\left(\left\{a_{1}, a_{2}\right\},\left\{a_{1}, a_{2}, a_{5}, a_{6}\right\}\right), \\
& F\left(\alpha_{2}\right)=\left(\left\{a_{2}, a_{3}, a_{4}\right\},\left\{a_{2}, a_{3}, a_{4}, a_{5}, a_{6}\right\}\right), \\
& F\left(\alpha_{3}\right)=\left(\left\{a_{5}, a_{7}, a_{8}\right\},\left\{a_{2}, a_{3}, a_{4}, a_{5}, a_{7}, a_{8}\right\}\right), \\
& F\left(\alpha_{4}\right)=\left(\left\{a_{1}, a_{2}, a_{3}, a_{4}, a_{5}\right\}, U\right) .
\end{aligned}
$$

Then $\pi\left(\alpha_{1}\right)=0.2, \pi\left(\alpha_{2}\right)=0.2, \pi\left(\alpha_{3}\right)=0.3, \pi\left(\alpha_{4}\right)=0.5$, Also $\pi^{\prime}\left(\alpha_{1}\right)=0.5, \pi^{\prime}\left(\alpha_{2}\right)=0.4$, $\pi^{\prime}\left(\alpha_{3}\right)=0.5, \pi^{\prime}\left(\alpha_{4}\right)=0.5$. Now, $A / \sim_{\pi}=\left\{\left\{\alpha_{1}, \alpha_{2}\right\},\left\{\alpha_{3}\right\},\left\{\alpha_{4}\right\}\right\}$ and $A / \sim_{\pi^{\prime}}=\left\{\left\{\alpha_{1}, \alpha_{3}, \alpha_{4}\right\},\left\{\alpha_{2}\right\}\right\}$.

Define a relation $\preceq$ on $A / \sim_{\pi}$ as the following

$$
[\alpha]_{\sim_{\pi}} \preceq[\beta]_{\sim_{\pi}} \text { if and only if } \pi(\alpha) \geq \pi(\beta) .
$$

Proposition 4.7. The relation $\preceq$ defined on $A / \sim_{\pi}$ is a total order.

Similarly define the relation $\preceq^{\prime}$ on $A / \sim_{\pi}$ as the following

$$
[\alpha]_{\sim_{\pi^{\prime}}} \preceq^{\prime}[\beta]_{\sim_{\pi^{\prime}}} \text { if and only } \pi^{\prime}(\alpha) \geq \pi^{\prime}(\beta) .
$$

Proposition 4.8. The relation $\preceq^{\prime}$ defined on $A / \sim_{\pi^{\prime}}$ is a total order. 


\subsection{Uncertainty measures for orthopair soft sets}

In this subsection idea of uncertainty measures for parameters is extended to the orthopair soft sets of first kind.

Definition 4.9. Let $(F, A) \in O S F(U)$. Then uncertainty index of $(F, A)$ is denoted by $\pi(F, A)$ and defined as

$$
\pi(F, A)= \begin{cases}1, & \text { if } A=\emptyset \\ \frac{1}{|A|} \sum_{\alpha \in A} \frac{|B N D(F(\alpha))|}{|U|}, & \text { otherwise. }\end{cases}
$$

When $A \neq \emptyset, \pi(F, A)=\frac{1}{|A|} \sum_{\alpha \in A} \frac{\left|Y_{F(\alpha)}-X_{F(\alpha)}\right|}{|U|}$.

Proposition 4.10. Let $(F, A) \in O S F(U)$. Then $0 \leq \pi(F, A) \leq 1$.

Definition 4.11. Let $(F, A) \in O S F(U)$. Then associated uncertainty index of $(F, A)$ is denoted by $\pi^{\prime}(F, A)$ and given by

$$
\pi^{\prime}(F, A)= \begin{cases}1, & \text { if } A=\emptyset, \\ \frac{1}{|A|} \sum_{\alpha \in A} \frac{|B N D(F(\alpha))|}{\left|Y_{F(\alpha)}\right|}, & \text { otherwise. }\end{cases}
$$

When $A \neq \emptyset, \pi^{\prime}(F, A)=\frac{1}{|A|} \sum_{\alpha \in A} \frac{\left|Y_{F(\alpha)}-X_{F(\alpha)}\right|}{\left|Y_{F(\alpha)}\right|}=\frac{1}{|A|} \sum_{\alpha \in A} \frac{\left|Y_{F(\alpha)} \cap X_{F(\alpha)}^{c}\right|}{\left|Y_{F(\alpha)}\right|}$.

Proposition 4.12. Let $(F, A) \in O S F(U)$. Then $\pi(F, A) \leq \pi^{\prime}(F, A) \leq 1$.

\section{$5 \quad$ Granularity of orthopair soft sets of first kind}

Granularity in orthopair soft sets is another source of uncertainty. When two or more objects satisfy a property associated with some parameter ( say $\alpha$ ), then these objects are indistinguishable from each other with respect to $\alpha$. In orthopair soft sets of first kind, various types of granularity can be defined. Nevertheless, we confine this study to three distinct types of mutually linked granularity.

Definition 5.1. Let $(F, A) \in O S F(U)$. Then lower granularity $G_{l}(F(\alpha))$ for any parameter $\alpha \in A$ is defined as $G_{l}(F(\alpha))=\frac{\left|X_{F(\alpha)}\right|}{|U|}$. Similarly, upper granularity $G_{u}(F(\alpha))$ is given by $G_{u}(F(\alpha))=\frac{\left|Y_{F(\alpha)}\right|}{|U|}$.

Granularity for $\alpha$ is the quantity $G(F(\alpha))=\frac{1}{2}\left(G_{l}(F(\alpha))+G_{u}(F(\alpha))\right)$.

Theorem 5.2. Let $(F, A) \in O S F(U)$. For any $\alpha \in A$, we have

$$
0 \leq G_{l}(F(\alpha)), G(F(\alpha)), G_{u}(F(\alpha)) \leq 1 .
$$

Theorem 5.3. Let $(F, A) \in O S F(U)$. Then the following hold:

1. $G_{l}(F(\alpha)) \leq G(F(\alpha)) \leq G_{u}(F(\alpha))$.

2. $G_{l}(F(\alpha) \cap F(\beta)) \leq \min \left(G_{l}(F(\alpha)), G_{l}(F(\beta))\right)$.

3. $G_{l}(F(\alpha) \cup F(\beta)) \geq \max \left(G_{l}(F(\alpha)), G_{l}(F(\beta))\right)$. 
4. $G_{u}(F(\alpha) \cap F(\beta)) \leq \min \left(G_{u}(F(\alpha)), G_{u}(F(\beta))\right)$.

5. $G_{u}(F(\alpha) \cup F(\beta)) \geq \max \left(G_{u}(F(\alpha)), G_{u}(F(\beta))\right)$.

6. $G(F(\alpha) \cap F(\beta)) \leq \min (G(F(\alpha)), G(F(\beta)))$.

7. $G(F(\alpha) \cup F(\beta)) \geq \max (G(F(\alpha)), G(F(\beta)))$.

Proof. (1) The proof of (1) follows from the definitions of $G_{l}, G$ and $G_{u}$.

(2) Consider

$$
\begin{aligned}
G_{l}(F(\alpha) \cap F(\beta)) & =\frac{\left|X_{F(\alpha) \cap F(\beta)}\right|}{|U|} \\
& \leq \min \left(\frac{\left|X_{F(\alpha)}\right|}{|U|}, \frac{\left|X_{F(\beta)}\right|}{|U|}\right) \\
& =\min \left(G_{l}(F(\alpha)), G_{l}(F(\beta))\right) .
\end{aligned}
$$

(3)

$$
\begin{aligned}
G_{l}(F(\alpha) \cup F(\beta)) & =\frac{\left|X_{F(\alpha) \cup F(\beta)}\right|}{|U|} \\
& \geq \max \left(\frac{\left|X_{F(\alpha)}\right|}{|U|}, \frac{\left|X_{F(\beta)}\right|}{|U|}\right) \\
& =\max \left(G_{l}(F(\alpha)), G_{l}(F(\beta))\right) .
\end{aligned}
$$

(4)

$$
\begin{aligned}
G_{u}(F(\alpha) \cap F(\beta)) & =\frac{\left|Y_{F(\alpha) \cap F(\beta)}\right|}{|U|} \\
& =\frac{\left|Y_{F(\alpha)} \cap Y_{F(\beta)}\right|}{|U|} \\
& \leq \min \left(\frac{\left|Y_{F(\alpha)}\right|}{|U|}, \frac{\left|Y_{F(\beta)}\right|}{|U|}\right) \\
& =\min \left(G_{u} F(\alpha), G_{u}(F(\beta))\right) .
\end{aligned}
$$

$(5)$

$$
\begin{aligned}
G_{u}(F(\alpha) \cup F(\beta)) & =\frac{\left|Y_{F(\alpha) \cup F(\beta)}\right|}{|U|} \\
& =\frac{\left|Y_{F(\alpha)} \cup Y_{F(\beta)}\right|}{|U|} \\
& \geq \max \left(\frac{\left|Y_{F(\alpha)}\right|}{|U|}, \frac{\left|Y_{F(\beta)}\right|}{|U|}\right) \\
& =\max \left(G_{U} F(\alpha), G_{U}(F(\beta))\right) .
\end{aligned}
$$

(6) It follows from (2) and (4).

(7) It follows from (3) and (5). 
It should be noted that equality does not hold in general in (2) to (7) of Theorem 5.3 as illustrated by the following example.

Example 5.4. (Continued from Example 4.0 ) It is easy to see that $G_{l}\left(F\left(\alpha_{1}\right)\right)=0.2, G_{l}\left(F\left(\alpha_{2}\right)\right)=$ 0.3. Now, $G_{l}\left(F\left(\alpha_{1}\right) \cap F\left(\alpha_{2}\right)\right)=0.1, G_{l}\left(F\left(\alpha_{1}\right) \cup F\left(\alpha_{2}\right)\right)=0.4$. Thus

$$
G_{l}\left(F\left(\alpha_{1}\right) \cap F\left(\alpha_{2}\right)\right)<\min \left(G_{l}\left(F\left(\alpha_{1}\right)\right), G_{l}\left(F\left(\alpha_{2}\right)\right)\right)
$$

and

$$
G_{l}\left(F\left(\alpha_{1}\right) \cup F\left(\alpha_{2}\right)\right)>\max \left(G_{l}\left(F\left(\alpha_{1}\right)\right), G_{l}\left(F\left(\alpha_{2}\right)\right)\right)
$$

Next, we have

$$
G_{u}\left(F\left(\alpha_{1}\right)\right)=0.4, G_{u}\left(F\left(\alpha_{2}\right)\right)=0.5, G_{u}\left(F\left(\alpha_{1}\right) \cap F\left(\alpha_{2}\right)\right)=0.3, \text { and } G_{u}\left(F\left(\alpha_{1}\right) \cup F\left(\alpha_{2}\right)\right)=0.6 .
$$

Hence,

$$
G_{u}\left(F\left(\alpha_{1}\right) \cap F\left(\alpha_{2}\right)\right)<\min \left(G_{u}\left(F\left(\alpha_{1}\right)\right), G_{u}\left(F\left(\alpha_{2}\right)\right)\right),
$$

and

$$
G_{u}\left(F\left(\alpha_{1}\right) \cup F\left(\alpha_{2}\right)\right)>\max \left(G_{u}\left(F\left(\alpha_{1}\right)\right), G_{u}\left(F\left(\alpha_{2}\right)\right)\right) .
$$

In addition,

$$
G\left(F\left(\alpha_{1}\right)\right)=0.3, G\left(F\left(\alpha_{2}\right)\right)=0.4, G\left(F\left(\alpha_{1}\right) \cap F\left(\alpha_{2}\right)\right)=0.2, \text { and } G\left(F\left(\alpha_{1}\right) \cup F\left(\alpha_{2}\right)\right)=0.5 .
$$

That is,

$$
G\left(F\left(\alpha_{1}\right) \cap F\left(\alpha_{2}\right)\right)<\min \left(G\left(F\left(\alpha_{1}\right)\right), G\left(F\left(\alpha_{2}\right)\right)\right)
$$

and

$$
G\left(F\left(\alpha_{1}\right) \cup F\left(\alpha_{2}\right)\right)>\max \left(G\left(F\left(\alpha_{1}\right)\right), G\left(F\left(\alpha_{2}\right)\right)\right) .
$$

Now the notion of granularities can be extended to the soft sets of first kind.

Definition 5.5. Let $(F, A) \in O S F(U)$. Lower granularity $G_{l}$ for $(F, A)$ is defined by

$$
G_{l}((F, A))=\frac{1}{|A|} \sum_{\alpha \in A} \frac{\left|X_{F(\alpha)}\right|}{|U|} .
$$

Upper granularity $G_{u}$ and mean granularity $G$ for $(F, A)$ are respectively defined as follows:

$$
\begin{gathered}
G_{u}((F, A))=\frac{1}{|A|} \sum_{\alpha \in A} \frac{\left|Y_{F(\alpha)}\right|}{|U|} ; \\
G(F, A)=\frac{1}{2}\left(G_{l}((F, A))+G_{u}((F, A))\right) .
\end{gathered}
$$

Proposition 5.6. Let $(F, A) \in O S F(U)$. Then we have

$$
G_{l}((F, A)) \leq G(F, A) \leq G_{u}((F, A)) .
$$

Example 5.7. (Continued from Example L.6) It is easy to see that $G_{l}\left(F\left(\alpha_{1}\right)\right)=0.2, G_{l}\left(F\left(\alpha_{2}\right)\right)=$ $0.3, G_{l}\left(F\left(\alpha_{3}\right)\right)=0.3, G_{l}\left(F\left(\alpha_{4}\right)\right)=0.5, G_{u}\left(F\left(\alpha_{1}\right)\right)=0.4, G_{u}\left(F\left(\alpha_{2}\right)\right)=0.5, G_{u}\left(F\left(\alpha_{3}\right)\right)=0.6$, $G_{u}\left(F\left(\alpha_{2}\right)\right)=1$. Then $G_{l}((F, A))=0.325, G_{u}((F, A))=0.625$ and $G(F, A)=0.475$. Therefore, we have

$$
G_{l}((F, A)) \leq G(F, A) \leq G_{u}((F, A)) .
$$




\section{$6 \quad$ Orthopair soft sets of second kind}

In Section 3, notion of orthopair soft sets of first kind has been studied. Now in this section concept of orthopair soft sets of second kind is being introduced. In a human society, it is natural to use terms like favour-against, like-dislike, positive-negative and friends-rivals. In every language such terminology is well understood. When such attributes/parameters/properties are used simultaneously to characterize elements of a certain set into two disjoint sets, then generally these two sets are not complement of each other. Orthopair soft sets of second kind provide an opportunity to represent and manipulate such information.

Definition 6.1. Let $E$ be the parameter space and $A \subseteq E$. An orthopair soft set of second kind $(F, A)$ over a set $U$ is defined by the map $F: A \rightarrow P(U) \times P(U)$ such that $F(\alpha)=(X, Y)$ and $X, Y \in P(U)$ and $X \cap Y=\emptyset$ for all $\alpha \in A$.

In case of orthopair soft sets of second kind, for each parameter, a pair of subsets of $U$ is obtained, where first subset is the collection of those elements which surely satisfy the property associated with the parameter and second subset is the collection of those elements which surely do not satisfy the property associated with the parameter.

An orthopair soft set $(F, A)$ of second kind over a set $U$ reduces to a soft set when $F(\alpha)=$ $(X, Y)$ and $Y=\emptyset$ for all $\alpha \in A$.

As for all $\alpha \in A, F(\alpha)=(X, Y)$, therefore each $F(\alpha)$ can be denoted by $\left(X_{F(\alpha)}, Y_{F(\alpha)}\right)$.

Throughout this paper $O S S(U)$ will denote the class of all orthopair soft set of second kind over $U$.

Definition 6.2. Let $(F, A) \in O S S(U)$. For any $\alpha \in A, F(\alpha)$ may be called a soft element of second kind for $(F, A)$.

Let $F(\alpha)=\left(X_{F(\alpha)}, Y_{F(\alpha)}\right)$ and $F(\beta)=\left(X_{F(\beta)}, Y_{F(\beta)}\right)$ be two soft elements of $(F, A) \in$ $O S S(U)$. Then $F(\alpha) \lesssim F(\beta)$ if and only if $X_{F(\alpha)} \subseteq X_{F(\beta)}$ and $Y_{F(\alpha)} \supseteq Y_{F(\beta)}$.

Let $F(\alpha)=(X, Y)$ be a soft element of second kind for $(F, A) \in O S S(U)$. Then $F^{c}(\alpha)=$ $\left(Y_{F(\alpha)}, X_{F(\alpha)}\right)$. HES $(F(\alpha))$ is a subset of $U$, defined as $\operatorname{HES}(F(\alpha))=U-\left(X_{F(\alpha)} \cup Y_{F(\alpha)}\right)$.

A soft set $\operatorname{HES}(F, A)$ over $U$, which is called hesitant soft set, might be useful for describing the hesitancy of orthopair soft sets $(F, A)$ of second kind.

Definition 6.3. Let $(F, A) \in O S S(U)$. Then $\operatorname{HES}(F, A)=(\operatorname{HES}(F), A)$ is a soft set defined as $\operatorname{HES}(F(\alpha))=U-\left(X_{F(\alpha)} \cup Y_{F(\alpha)}\right)=\left(X_{F(\alpha)} \cup Y_{F(\alpha)}\right)^{c}=X_{F(\alpha)}^{c} \cap Y_{F(\alpha)}^{c}$ for all $\alpha \in A$.

Orthopair soft sets of second kind are very useful to accommodate and represent conflicting points of views about the elements of a universal set. For example in professional organizations/societies it is customary, for different positions elections are held among the panels of individuals. Orthopair soft sets of second kind not only show the votes in favor and against an individual candidate but also show the position of whole panel. Moreover the associated hesitant soft set describes the voters who do not want to participate in the election process for a particular candidate.

Example 6.4. Let the electoral college of a professional organization be the set $U=\left\{x_{1}, x_{2}, \ldots, x_{20}\right\}$ and set $A=\left\{\alpha_{1}, \alpha_{2}, \alpha_{3}, \alpha_{4}\right\}$ be the set of members of a panel of candidates, where $\alpha_{1}, \alpha_{2}, \alpha_{3}, \alpha_{4}$ represent president, vice president, secretary and information secretary, respectively. The orthopair soft set $(F, A)$ of second kind over $U$, represents the votes in favor and against each candidate of 
the panel as the following.

$$
\begin{aligned}
& F\left(\alpha_{1}\right)=\left(\left\{x_{1}, x_{2}, x_{7}, x_{9}, x_{11}, x_{12}, x_{15}, x_{20}\right\},\left\{x_{3}, x_{5}, x_{8}, x_{13}, x_{19}\right\}\right) \\
& F\left(\alpha_{2}\right)=\left(\left\{x_{1}, x_{2}, x_{4}, x_{6}, x_{7}, x_{9}, x_{11}, x_{12}, x_{15}, x_{20}\right\},\left\{x_{3}, x_{5}, x_{8}, x_{10}, x_{13}, x_{16}, x_{19}\right\}\right) \\
& F\left(\alpha_{3}\right)=\left(\left\{x_{1}, x_{2}, x_{4}, x_{6}, x_{7}, x_{9}, x_{10}, x_{11}, x_{12}, x_{15}, x_{16}, x_{20}\right\},\left\{x_{3}, x_{5}, x_{8}, x_{13}, x_{19}\right\}\right) \\
& F\left(\alpha_{4}\right)=\left(\left\{x_{1}, x_{2}, x_{3}, x_{7}, x_{9}, x_{11}, x_{12}, x_{15}, x_{19}\right\},\left\{x_{5}, x_{8}, x_{13}, x_{20}\right\}\right) .
\end{aligned}
$$

Now, the soft set $H E S(F, A)$ is given as the following

$$
\begin{aligned}
& \operatorname{HES}\left(F\left(\alpha_{1}\right)\right)=\left\{x_{4}, x_{6}, x_{10}, x_{14}, x_{16}, x_{17}, x_{18}\right\} \\
& \operatorname{HES}\left(F\left(\alpha_{2}\right)\right)=\left\{x_{14}, x_{17}, x_{18}\right\} \\
& \operatorname{HES}\left(F\left(\alpha_{3}\right)\right)=\left\{x_{14}, x_{17}, x_{18}\right\} \\
& \operatorname{HES}\left(F\left(\alpha_{4}\right)\right)=\left\{x_{4}, x_{6}, x_{10}, x_{14}, x_{17}, x_{18}\right\}
\end{aligned}
$$

Definition 6.5. Let $(F, A),(G, B) \in O S S(U)$. Then $(F, A)$ is soft contained in $(G, B)$ if the following hold:

1. $A \subseteq B$.

2. $F(\alpha) \lesssim G(\alpha)$ for all $\alpha \in A$.

If $(F, A)$ is soft contained in $(G, B)$, then it will be denoted by $(F, A) \widetilde{\subseteq}(G, B)$. If $(F, A) \widetilde{\subseteq}(G, B)$ and $(G, B) \widetilde{\subseteq}(F, A)$, then $(F, A)=(G, B)$.

Definition 6.6. Let $(F, A) \in O S S(U)$. Then the complement of $(F, A)$ is denoted by $(F, A)^{c}=$ $\left(F^{c}, A\right)$ and is defined as $F^{c}(\alpha)=\left(Y_{F(\alpha)}, X_{F(\alpha)}\right)$, where $F(\alpha)=(X, Y)$ for all $\alpha \in A$.

It is easy to see that $\left((F, A)^{c}\right)^{c}=(F, A)$.

Definition 6.7. Let $(F, A) \in O S S(U)$. Then we define:

1. $\square(F, A)=(\square F, A)$ is an orthopair soft set of second kind defined as $\square F(\alpha)=\left(X_{F(\alpha)}, X_{F(\alpha)}^{c}\right)$, where $F(\alpha)=(X, Y)$ for all $\alpha \in A$.

2. $\diamond(F, A)=(\diamond F, A)$ is an orthopair soft set of second kind defined as $\diamond F(\alpha)=\left(Y_{F(\alpha)}, Y_{F(\alpha)}^{c}\right)$, where $F(\alpha)=(X, Y)$ for all $\alpha \in A$.

Definition 6.8. Let $(F, A),(G, B) \in O S S(U)$. Then we define:

1. their restricted intersection $(F, A) \cap_{R}(G, B)$ is defined as

$$
(F, A) \cap_{R}(G, B)=(K, C) \text {, where } C=A \cap B .
$$

and when $C \neq \emptyset$

$$
K(\alpha)=\left(X_{F(\alpha)} \cap X_{G(\alpha)}, Y_{F(\alpha)} \cup Y_{G(\alpha)}\right) \text { for all } \alpha \in C
$$

otherwise $(K, C)=\emptyset_{\emptyset}$. 
2. their extended intersection $(F, A) \cap_{\mathcal{E}}(G, B)$ is defined as

$$
(F, A) \cap_{\mathcal{E}}(G, B)=(K, C) \text {, where } C=A \cup B .
$$

and

$$
K(\alpha)=\left\{\begin{array}{cc}
\left(X_{F(\alpha)}, Y_{F(\alpha)}\right) & \text { if } \alpha \in A-B \\
\left(X_{G(\alpha)}, Y_{G(\alpha)}\right) & \text { if } \alpha \in B-A \\
\left(X_{F(\alpha)} \cap X_{G(\alpha)}, Y_{F(\alpha)} \cup Y_{G(\alpha)}\right) & \text { if } \alpha \in A \cap B
\end{array}\right.
$$

3. their restricted union $(F, A) \cup_{R}(G, B)$ is defined as

$$
(F, A) \cup_{R}(G, B)=(K, C) \text {, where } C=A \cap B .
$$

and when $C \neq \emptyset$

$$
K(\alpha)=\left(X_{F(\alpha)} \cup X_{G(\alpha)}, Y_{F(\alpha)} \cap Y_{G(\alpha)}\right) \text { for all } \alpha \in C
$$

otherwise $(K, C)=\emptyset_{\emptyset}$.

4. their extended union $(F, A) \cup_{\mathcal{E}}(G, B)$ is defined as

$$
(F, A) \cup_{\mathcal{E}}(G, B)=(K, C) \text {, where } C=A \cup B .
$$

and

$$
K(\alpha)=\left\{\begin{array}{cc}
\left(X_{F(\alpha)}, Y_{F(\alpha)}\right) & \text { if } \alpha \in A-B \\
\left(X_{G(\alpha)}, Y_{G(\alpha)}\right) & \text { if } \alpha \in B-A \\
\left(X_{F(\alpha)} \cup X_{G(\alpha)}, Y_{F(\alpha)} \cap Y_{G(\alpha)}\right) & \text { if } \alpha \in A \cap B
\end{array}\right.
$$

Theorem 6.9. Let $(F, A),(G, B),(H, C) \in O S S(U)$. Then we have

$$
((F, A) \theta(G, B)) \theta(H, C)=(F, A) \theta((G, B) \theta(H, C)),
$$

where $\theta \in\left\{\cap_{R}, \cup_{R}, \cap_{\mathcal{E}}, \cup_{\mathcal{E}}\right\}$.

Theorem 6.10. Let $(F, A),(G, B) \in O S S(U)$. Then the following hold:

1. $\left((F, A) \cap_{R}(G, B)\right)^{c}=(F, A)^{c} \cup_{R}(G, B)^{c}$.

2. $\left((F, A) \cup_{R}(G, B)\right)^{c}=(F, A)^{c} \cap_{R}(G, B)^{c}$.

Proof. (1) If $A \cap B=\emptyset$, then equality holds trivially. Let $A \cap B \neq \emptyset$ and $(F, A) \cap_{B}(G, B)=(H, C)$, where $C=A \cap B$ and $H(\alpha)=F(\alpha) \cap G(\alpha)$ for all $\alpha \in C$. As $\left((F, A) \cap_{R}(G, B)\right)^{c}=(H, C)^{c}=$ $\left(H^{c}, C\right)$. Where

$$
\begin{aligned}
H^{c}(\alpha) & =(F(\alpha) \cap G(\alpha))^{c} \text { for all } \alpha \in C \\
& =\left(X_{F(\alpha)} \cap X_{G(\beta)}, Y_{F(\alpha)} \cup Y_{G(\beta)}\right)^{c} \\
& =\left(Y_{F(\alpha)} \cup Y_{G(\beta)}, X_{F(\alpha)} \cap X_{G(\beta)}\right)
\end{aligned}
$$

On the other hand let $(F, A)^{c} \cup_{R}(G, B)^{c}=(K, C)$. This implies $(K, C)=\left(F^{c}, A\right) \cup_{R}\left(G^{c}, B\right)$. Now for all $\alpha \in C$,

$$
\begin{aligned}
K(\alpha) & =F^{c}(\alpha) \cup G^{c}(\alpha) \\
& =\left(Y_{F(\alpha)}, X_{F(\alpha)}\right) \cup\left(Y_{G(\beta)}, X_{G(\beta)}\right) \\
& =\left(Y_{F(\alpha)} \cup Y_{G(\beta)}, X_{F(\alpha)} \cap X_{G(\beta)}\right) .
\end{aligned}
$$

This shows that for all $\alpha \in C, H^{c}(\alpha)=K(\alpha)$. Hence

$$
\left((F, A) \cap_{R}(G, B)\right)^{c}=(F, A)^{c} \cup_{R}(G, B)^{c} .
$$

(2) The proof follows from (1) and thus omitted. 


\section{$7 \quad$ Uncertainty measures for orthopair soft sets of second kind}

In orthopair soft sets of second kind, hesitancy is a major reason for uncertainty. In the following uncertainty index for the parameters is studied.

Definition 7.1. Let $(F, A) \in O S S(U)$. For each $\alpha \in A$, an uncertainty measure called uncertainty index for the parameter $\alpha$ denoted by $\pi(\alpha)$ can be defined as

$$
\pi(\alpha)=\frac{|H E S(F(\alpha))|}{|U|}=\frac{\left|X_{F(\alpha)}^{c} \cap Y_{F(\alpha)}^{c}\right|}{|U|} .
$$

It is clear that $0 \leq \pi(\alpha) \leq 1$.

If for some $\alpha \in A, F(\alpha)=(\emptyset, \emptyset)$, then $\pi(\alpha)=1$. On the other hand when $F(\alpha)=\left(X, X^{c}\right)$, then $\pi(\alpha)=0$. It has been seen already that in case of orthopair soft sets of first kind uncertainty measures give rise to an equivalence relation on the set of parameters. Similarly in orthopair soft sets of second kind above defined uncertainty measure again induces an equivalence relation on the set of parameters.

Definition 7.2. Let $(F, A) \in O S S(U)$. For any $\alpha, \beta \in A$, define a relation $\bar{\sim}_{\pi}$

$$
\alpha \bar{\sim}_{\pi} \beta \text { if and only if } \pi(\alpha)=\pi(\beta) .
$$

Proposition 7.3. Let $(F, A) \in O S S(U)$. Then the relation $\bar{\sim}_{\pi}$ is an equivalence relation on $A$.

Let $(F, A) \in O S S(U)$. Then we can define $A / \bar{\sim}_{\pi}=\left\{[\alpha]_{\bar{\sim}_{\pi}}: \alpha \in A\right\}$, where $[\alpha]_{\bar{\sim}_{\pi}}$ is the equivalence class containing $\alpha \in A$.

Define a relation $\preceq$ on $A / \bar{\sim}_{\pi}$ as the following

$$
[\alpha]_{\bar{\sim}_{\pi}} \preceq[\beta]_{\bar{\sim}_{\pi}} \text { if and only } \pi(\alpha) \geq \pi(\beta) .
$$

Proposition 7.4. The relation $\preceq$ defined on $A / \bar{\sim}_{\pi}$ is a total order.

Definition 7.5. Let $(F, A)$ be an orthopair soft set of second kind over $U$. Then uncertainty index of $(F, A)$ is denoted by $\pi(F, A)$ and defined as

$$
\pi(F, A)= \begin{cases}1, & \text { if } A=\emptyset \\ \frac{1}{|A|} \sum_{\alpha \in A} \frac{|H E S(F(\alpha))|}{|U|}, & \text { otherwise. }\end{cases}
$$

When $A \neq \emptyset, \pi(F, A)=\frac{1}{|A|} \sum_{\alpha \in A} \frac{\left|X_{F(\alpha)}^{c} \cap Y_{F(\alpha)}^{c}\right|}{|U|}$.

Proposition 7.6. Let $(F, A) \in O S S(U)$. Then $0 \leq \pi(F, A) \leq 1$.

Proposition 7.7. Let $(F, A) \in O S S(U)$. Then

1. $\pi(\square(F, A))=0$.

2. $\pi(\diamond(F, A))=0$. 


\section{Granularity of orthopair soft sets of second kind}

Different granularity measures for orthopair soft sets of first kind has been discussed in Section 1 . Here these concept takes a new forms in a natural way when orthopair soft sets of second kind are being considered.

Definition 8.1. Let $(F, A) \in O S S(U)$. Membership granularity $G_{m}(F(\alpha))$ for any parameter $\alpha \in A$ is defined as $G_{m}(F(\alpha))=\frac{\left|X_{F(\alpha)}\right|}{|U|}$. Similarly, nonmembership granularity $G_{n}(F(\alpha))$ is given by $G_{n}(F(\alpha))=\frac{\left|Y_{F(\alpha)}\right|}{|U|}$.

Granularity for $\alpha$ is the quantity $G(F(\alpha))=\frac{1}{2}\left(G_{m}(F(\alpha))+G_{n}(F(\alpha))\right)$.

Theorem 8.2. Let $(F, A) \in O S S(U)$. For any $\alpha \in A$, we have

$$
0 \leq G_{m}(F(\alpha)), G(F(\alpha)), G_{n}(F(\alpha)) \leq 1 .
$$

Theorem 8.3. Let $(F, A) \in O S S(U)$. Then any $\alpha, \beta \in A$, the following hold:

1. $G_{m}(F(\alpha) \cap F(\beta)) \leq \min \left(G_{m}(F(\alpha)), G_{m}(F(\beta))\right)$.

2. $G_{m}(F(\alpha) \cup F(\beta)) \geq \max \left(G_{m}(F(\alpha)), G_{m}(F(\beta))\right)$.

3. $G_{n}(F(\alpha) \cap F(\beta)) \geq \max \left(G_{n}(F(\alpha)), G_{n}(F(\beta))\right)$.

4. $G_{n}(F(\alpha) \cup F(\beta)) \leq \min \left(G_{n}(F(\alpha)), G_{n}(F(\beta))\right)$.

Proof. (1) Consider

$$
\begin{aligned}
G_{m}(F(\alpha) \cap F(\beta)) & =\frac{\left|X_{F(\alpha) \cap F(\beta)}\right|}{|U|} \\
& \leq \min \left(\frac{\left|X_{F(\alpha)}\right|}{|U|}, \frac{\left|X_{F(\beta)}\right|}{|U|}\right) \\
& =\min \left(G_{m}(F(\alpha)), G_{m}(F(\beta))\right) .
\end{aligned}
$$

$$
\begin{aligned}
G_{m}(F(\alpha) \cup F(\beta)) & =\frac{\left|X_{F(\alpha) \cup F(\beta)}\right|}{|U|} \\
& \geq \max \left(\frac{\left|X_{F(\alpha)}\right|}{|U|}, \frac{\left|X_{F(\beta)}\right|}{|U|}\right) \\
& =\max \left(G_{m}(F(\alpha)), G_{m}(F(\beta))\right) .
\end{aligned}
$$

$$
\begin{aligned}
G_{n}(F(\alpha) \cap F(\beta)) & =\frac{\left|Y_{F(\alpha) \cup F(\beta)}\right|}{|U|} \\
& =\frac{\left|Y_{F(\alpha)} \cup Y_{F(\beta)}\right|}{|U|} \\
& \geq \max \left(\frac{\left|Y_{F(\alpha)}\right|}{|U|}, \frac{\left|Y_{F(\beta)}\right|}{|U|}\right) \\
& =\max \left(G_{n} F(\alpha), G_{n}(F(\beta))\right) .
\end{aligned}
$$


(4)

$$
\begin{aligned}
G_{n}(F(\alpha) \cup F(\beta)) & =\frac{\left|Y_{F(\alpha) \cap F(\beta)}\right|}{|U|} \\
& =\frac{\left|Y_{F(\alpha)} \cap Y_{F(\beta)}\right|}{|U|} \\
& \leq \min \left(\frac{\left|Y_{F(\alpha)}\right|}{|U|}, \frac{\left|Y_{F(\beta)}\right|}{|U|}\right) \\
& =\min \left(G_{n} F(\alpha), G_{n}(F(\beta))\right) .
\end{aligned}
$$

Next the concept of granularities can be extended to the soft sets of second kind.

Definition 8.4. Let $(F, A) \in O S S(U)$. Membership granularity of $(F, A)$ is denoted and defined by $G_{m}((F, A))=\frac{1}{|A|} \sum_{\alpha \in A} \frac{\left|X_{F(\alpha)}\right|}{|U|}$.

In a similar fashion, nonmembership granularity $G_{n}$ and mean granularity $G$ for $(F, A)$ are defined as follows:

$$
\begin{aligned}
G_{n}((F, A)) & =\frac{1}{|A|} \sum_{\alpha \in A} \frac{\left|Y_{F(\alpha)}\right|}{|U|} \\
G(F, A) & =\frac{1}{2}\left(G_{m}((F, A))+G_{n}((F, A))\right) .
\end{aligned}
$$

Proposition 8.5. Let $(F, A) \in O S S(U)$. Then we have

$$
0 \leq G_{m}((F, A)), G(F, A), G_{n}((F, A)) \leq 1
$$

\section{$9 \quad$ Decision making based on orthopair soft sets}

In human life collective wisdom helps to take important decisions based on consensus. In the following an example is given where decision making based on orthopair soft sets of second kind is considered.

Example 9.1. Let there be three fund managers $X, Y$ and $Z$ to look after the interest of a mutual fund management company. Let $U=\left\{c_{1}, c_{2}, \ldots, c_{20}\right\}$ be the set of companies in different sectors which they are going to consider on a particular day for investment. Let $A=\left\{\alpha_{1}, \alpha_{2}, \alpha_{3}, \alpha_{4}\right\}$ be the set of parameters to denote the sectors where above mentioned companies do business. Consider $\alpha_{1}, \alpha_{2}, \alpha_{3}$ and $\alpha_{4}$ denote banking, automobile, energy and hi-tech sectors, respectively. Initially, they construct orthopair soft sets of second kind to depict where they want to buy and not to buy stocks. Then they consider restricted intersection of these orthopair soft sets of second kind to have a decision based on consensus. Let $F, G, H$ be mappings from set $A$ to $P(U) \times P(U)$. Let the following orthopair soft sets $(F, A),(G, A)$ and $(H, A)$ are obtained by fund managers $X, Y$ and $Z$, respectively.

$$
\begin{aligned}
(F, A) & =\left\{F\left(\alpha_{1}\right)=\left(\left\{c_{1}, c_{3}\right\},\left\{c_{2}, c_{4}, c_{5}\right\}\right), F\left(\alpha_{2}\right)=\left(\left\{c_{9}, c_{10}\right\},\left\{c_{6}, c_{8}\right\}\right),\right. \\
F\left(\alpha_{3}\right) & \left.=\left(\left\{c_{12}, c_{14}\right\},\left\{c_{11}, c_{13}, c_{15}\right\}\right), F\left(\alpha_{4}\right)=\left(\left\{c_{18}, c_{19}\right\},\left\{c_{16}, c_{20}\right\}\right)\right\}
\end{aligned}
$$




$$
\begin{aligned}
&(G, A)=\left\{G\left(\alpha_{1}\right)=\left(\left\{c_{1}, c_{4}\right\},\left\{c_{2}, c_{3}, c_{5}\right\}\right), G\left(\alpha_{2}\right)=\left(\left\{c_{6}, c_{10}\right\},\left\{c_{7}, c_{8}, c_{9}\right\}\right),\right. \\
& G\left(\alpha_{3}\right)=\left.\left.\left(\left\{c_{11}, c_{15}\right\},\left\{c_{12}, c_{13}\right\}\right), G\left(\alpha_{4}\right)=\left(\left\{c_{18}, c_{19}\right\},\left\{c_{20}\right\}\right)\right)\right\} ; \\
&(H, A)=\left\{H\left(\alpha_{1}\right)=\left(\left\{c_{1}, c_{3}\right\},\left\{c_{2}, c_{4}\right\}\right), H\left(\alpha_{2}\right)=\left(\left\{c_{10}\right\},\left\{c_{6}, c_{8}, c_{9}\right\}\right),\right. \\
&\left.H\left(\alpha_{3}\right)=\left(\left\{c_{13}, c_{14}\right\},\left\{c_{11}, c_{12}, c_{15}\right\}\right), G\left(\alpha_{4}\right)=\left(\left\{c_{18}, c_{19}\right\},\left\{c_{16}, c_{20}\right\}\right)\right\} .
\end{aligned}
$$

Restricted intersection $(K, A)$ of these three orthopair soft sets of second kind is obtained as follows:

$$
\begin{aligned}
& (K, A)=\left\{K\left(\alpha_{1}\right)=\left(\left\{c_{1}\right\},\left\{c_{2}, c_{3}, c_{4}, c_{5}\right\}\right), K\left(\alpha_{2}\right)=\left(\left\{c_{10}\right\},\left\{c_{6}, c_{7}, c_{8}, c_{9}\right\}\right)\right. \\
& \left.K\left(\alpha_{3}\right)=\left(\emptyset,\left\{c_{11}, c_{12}, c_{13}, c_{15}\right\}\right), K\left(\alpha_{4}\right)=\left(\left\{c_{18}, c_{19}\right\},\left\{c_{16}, c_{20}\right\}\right)\right\} .
\end{aligned}
$$

The orthopair soft set $(K, A)$ of second kind determines that in banking sector company $c_{1}$ will be surely in profit whereas companies $c_{2}, c_{3}, c_{4}, c_{5}$ are not good choices for the investment. Similarly conclusions can be drawn for the companies working in other sectors.

\section{Conclusions}

The notion of orthopair soft sets has been introduced in this paper, which provides a mathematical frame work to handle uncertainty attached to elements of a subset when some parameters are used for their description. For this purpose, two different kinds of orthopair soft sets have been discussed. In both types of orthopair soft sets, there are particular type of uncertainties linked with each parameter. This helps us to classify elements of the set of parameters. Granularity is another source of uncertainty associated with elements of a set. Granularity measures for both types of orthopair soft sets has been discussed. In addition, an example has been presented to illustrate possible application of orthopair soft sets to decision making problems.

\section{Acknowledgments}

This work was supported in part by the National Natural Science Foundation of China (Grant No. 11301415), and in part by the Natural Science Basic Research Plan in Shaanxi Province of China (Grant No. 2018JM1054).

\section{References}

[1] M. Agarwal, K.K. Biswas, M. Hanmandlu, Generalized intuitionistic fuzzy soft sets with applications in decision-making, Applied Soft Computing, 13 (2013), 3552-3566.

[2] M.I. Ali, Another view on q-rung orthopair fuzzy sets, International Journal of Intelligent Systems, 33 (2018), 2139-2153.

[3] A. Ali, M.I. Ali, N. Rehman, Soft dominance based rough sets with applications in information systems, International Journal of Approximate Reasoning, 113 (2019), 171-195.

[4] M.I. Ali, B. Davvaz, M. Shabir, Generalized fuzzy S-acts and their characterization by soft S-acts, Neural Computing and Applications, 21(1) (2012), 9-17.

[5] M.I. Ali, F. Feng, X.Y. Liu, W.K. Min, M. Shabir, On some new operations in soft set theory, Computers and Mathematics with Applications, 57 (2009), 1547-1553. 
[6] M.I. Ali, N. Mehmood, J.M. Zhan, N. Shah, Soft linear programming: An application of soft vector spaces, Journal of Information and Optimization Sciences, 41(3) (2020), 679-704.

[7] M.I. Ali, M. Shabir, M. Naz, Algebraic structures of soft sets associated with new operations, Computers and Mathematics with Applications, 61 (2011), 2647-2654.

[8] K. Atanassov, Intuitionistic fuzzy sets, Fuzzy Sets and Systems, 20 (1986), 87-96.

[9] K. Atanassov, Intuitionistic fuzzy sets, Springer, Heidelberg, 1999.

[10] D. Ciucci, Orthopairs: A simple and widely used way to model uncertainty, Fundamenta Informaticae, 108 (2011), 287-304.

[11] D. Dubois, H. Prade, Two-fold fuzzy sets and rough sets-some issues in knowledge representation, Fuzzy Sets and Systems, 23 (1987), 3-18.

[12] D. Dubois, H. Prade, An introduction to bipolar representation of information and preference, International Journal of Intelligent Systems, 23 (2008), 866-877.

[13] F. Feng, H. Fujita, M.I. Ali, R.R. Yager, X. Liu, Another view on generalized intuitionistic fuzzy soft sets and related multiattribute decision making methods, IEEE Transactions on Fuzzy Systems, 27(3) (2019), 474-488.

[14] F. Feng, Z. Xu, H. Fujita, M. Liang, Enhancing PROMETHEE method with intuitionistic fuzzy soft sets, International Journal of Intelligent Systems, 35 (2020), 1071-1104.

[15] F. Feng, Y. Zheng, B. Sun, M. Akram, Novel score functions of generalized orthopair fuzzy membership grades with application to multiple attribute decision making, Granular Computing, (2021), https://doi.org/10.1007/s41066-021-00253-7.

[16] M.Y. Gentilhomme, Les ensembles flous en linguistique, Cahiers de Linguistique Theoretique et Applique, Bucarest, 47 (1968), 47-65.

[17] P.K. Maji, R. Biswas, A.R. Roy, Intuitionistic fuzzy soft sets, Journal of Fuzzy Mathematics, $9(3)$ (2001), 677-692.

[18] D. Molodtsov, Soft set theory-first results, Computers and Mathematics with Applications, 37 (1999), 19-31.

[19] M. Naz, M. Shabir, On fuzzy bipolar soft sets, their algebraic structures and applications, Journal of Intelligent and Fuzzy Systems, 26(4) (2014), 1645-1656.

[20] Z. Pawlak, Rough sets, International Journal of Computer and Information Sciences, 11 (1982), 341-356.

[21] Z. Pawlak, Rough sets-theoretical aspects of reasoning about data, Kluwer Academic Publisher, 1991.

[22] W. Pedrycz, Shadowed sets: Representing and processing fuzzy sets, IEEE Transaction on Systems, Man and Cybernetics-PART B: Cybernetics, 28(1) (1998), 103-109.

[23] W. Pedrycz, Shadowed sets: Bridging fuzzy and rough sets, in: Rough Fuzzy Hybridization (S. Pal, A. Skowron, Eds.), Springer-Verlag, Singapore, (1999), 179-199. 
[24] W. Pedrycz, G. Vukovich, Granular computing with shadowed sets, International Journal of Intelligent Systems, 17 (2002), 173-197.

[25] N. Rehman, A. Ali, M.I. Ali, C. Park, SDMGRS: Soft dominance based multi granulation rough sets and their applications in conflict analysis problems, IEEE Access, 6 (2018), 3139931416.

[26] R.R. Yager, Pythagorean membership grades in multi-criteria decision making, Technical Report MII-3301. New Rochelle, NY: Machine Intelligence Institute, Iona College, 2013.

[27] R.R. Yager, Pythagorean membership grades in multi-criteria decision making, IEEE Transactions on Fuzzy Systems, 22 (2014), 958-965.

[28] R.R. Yager, Generalized orthopair fuzzy sets, IEEE Transactions on Fuzzy Systems, 25 (2017), $1222-1230$.

[29] R.R. Yager, A. M. Abbasov, Pythagorean membership grades, complex numbers, and decision making, International Journal of Intelligent Systems, 28 (2013), 436-452.

[30] Y.Y. Yao, X. Li, Comparison of rough-set and interval-set models for uncertain reasoning, Fundamenta Informaticae, 27 (1997), 289-298.

[31] L.A. Zadeh, Fuzzy sets, Information and Control, 8 (1965), 338-353.

[32] W.R. Zhang, Bipolar fuzzy sets and relations: A computational framework for cognitive modeling and multiagent decision analysis, Proceedings of the First International Joint Conference of The North American Fuzzy Information Processing Society Biannual Conference, (1994), 305-309. 